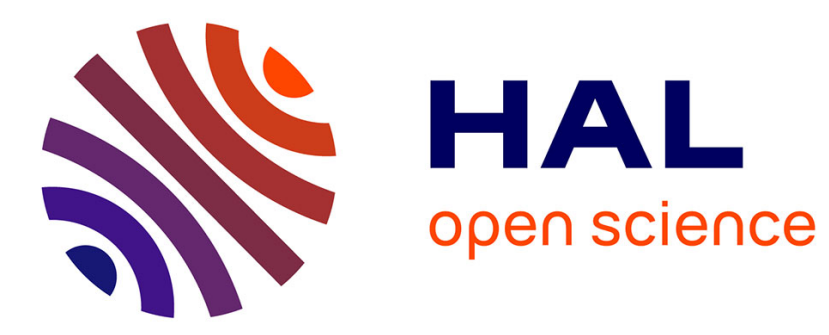

\title{
Note sur les propriétés des contacts imparfaits. Travaux de M. Fisch, M. Schneider, M. Blanc \\ Pierre Weiss
}

\section{To cite this version:}

Pierre Weiss. Note sur les propriétés des contacts imparfaits. Travaux de M. Fisch, M. Schneider, M. Blanc. J. Phys. Theor. Appl., 1906, 5 (1), pp.462-467. 10.1051/jphystap:019060050046201 . jpa-00241128

\section{HAL Id: jpa-00241128 https://hal.science/jpa-00241128}

Submitted on 1 Jan 1906

HAL is a multi-disciplinary open access archive for the deposit and dissemination of scientific research documents, whether they are published or not. The documents may come from teaching and research institutions in France or abroad, or from public or private research centers.
L'archive ouverte pluridisciplinaire HAL, est destinée au dépôt et à la diffusion de documents scientifiques de niveau recherche, publiés ou non, émanant des établissements d'enseignement et de recherche français ou étrangers, des laboratoires publics ou privés. 


\section{NOTE SUR LES PROPRIÉTÉS DES CONTACTS IMPARFAITS. TRAVAUX DE M. FISCH, M. SCHNEIDER, M. BLANC; \\ Par M. Pierle Weiss.}

Le travail de M. Fisch est uniquement de nature expérimentale et descriptive. Au moment où il a été entrepris, plusieurs physiciens, MM. Guthe et Trowbridge, M. Ph.-E. Robinson, avaient déjà substitué dans des recherches analogues, à l'excitation du cohéreur par les ondes, difficileà obtenir identique à elle-même et à mesurer, l'excitation du cohéreur par un courant continu traversant le contact. L'analogie profonde, sinon l'identité, de ces deux modes d'excitation, n'est pas douteuse. Le résultat saillant de ces recherches était dans la caractéristique, c'est-à-dire dans la courbe représentant pour cet appareil la

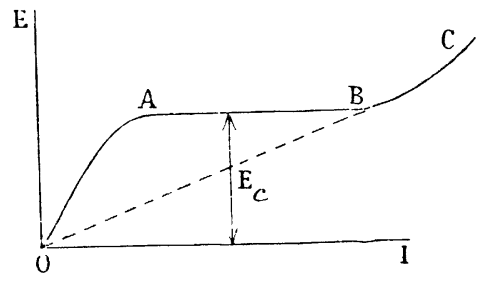

FIG. 1.

force électromotrice en fonction de l'intensité du courant, l'existence d'une portion horizontale étendue $\Lambda \mathrm{B}$, fí, 1). Le long de $\mathrm{AB}$ la résistance du contact, donnée par le coefficient angulaire de la droite qui va d'un point de la courbe à l'origine, décroît régulièrement. $\mathrm{AB}$ est en quelque sorte l'image graphique de la cohération. Guthe et Trowlsridgee ont donné à la force électromotrice correspondant à $\mathrm{AB}$ le nom de terexion critique $\left(\mathrm{E}_{c}\right.$.

On pouvait se demander si cette tension critique était une cons- 
tante naturelle et quelle etait sa grandeur dans diverses circonstances.

Après avoir constaté. par de nombreuses expériences. que le courant alternatif et le courant 'ontinu a sissent de la minte façon, avec une plus grande sensibilité au courant alternatif, que la différence entre la force électromotrice maxima "t la force électromotrice efticace ne suffit pas a expliquer, M. Fisch s... .11!. de cette tension critique.

11 montre que la région $A B$ est très inégalement développée suivant les conditions de l'expérience. Tandis que dans certaines séries d’xpériences se rapportant à des surfaces de fer plon xées dans laair la trusion oscille dans le voisinage immédiat de 1.21 volt, l’intensité du courant variait de 6 à 300 milliampères, c'est-à-dire dans le rapport de 1 à 50 ; lorsque ces mêmes surfaces sont plongiés dans d'autres milieux : l'alcool, l'huile de paraffine, la wly lint. le phenomène perd de sa netteté, et ce n'est plus que par à peu près que l'on peut déduire des courbes la valeur numérique d'une tension jouant le rôle de tension critique. Dans tous les cas la courbe, après la portion $\mathrm{AB}$, se continue par une portion ascendante $\mathrm{BC}$.

M. Fisch indique pour la tension critique d'un cohéreur de fer dans:

\begin{tabular}{|c|c|}
\hline L’air. . & $0,2,2$ \\
\hline Le pélrole.. & $(1,2) \cdot 2$ \\
\hline La térébenthine & 0.21 \\
\hline L'alcoul...... & $0,20 \ddot{3}$ \\
\hline I'eau... & 0,20 \\
\hline Lhuile de paltaltine. & 0.29 \\
\hline La glycérin'........ & 0,78 \\
\hline
\end{tabular}

Cette tension critique semble donc dépendre surtout du solide et beaucoup moins du milieu interpose. Cela renth tes cinq premines nombres. Les deux dernitre mombren montrent que cert.

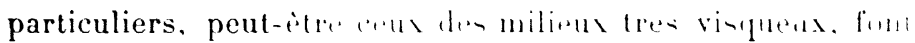

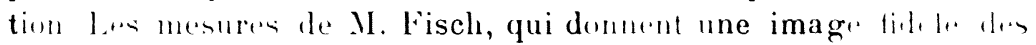
proprietés expérimentales du cohéreur, laissent donc en detinitive l'impression que celte tension critique est une résultante complexe de plusieurs facteurs et ne font pas avancer la connai $\ldots \ldots \ldots$ le la nature intime du phénomène.

M. Schneider au cours dune elme experimentale sur la mime

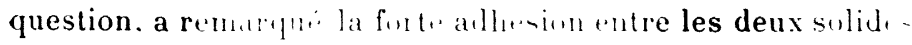


tact. après la cohération. Il a cru trouver dans cette circonstance un moyen d’analyse du phénomìne et a été conduit à faire l'hypothèse qui consiste à admettre la soudure par fusion des arêtes vives ou angles proéminents par lesquels les corps se touchent. Son travail consiste donc à rechercher jusqu'à quel point cette hypothèse est conforme aux faits.

La forme de la courbe représentant la relation entre $\mathrm{E}$ et $\mathrm{I}$ s’explique aisément dans cette hypothèse. E tend à croître par suite de la loi d'Ohm, mais en même temps E décroît au fur et à mesure de l'augmentation de la surface de contact par la fusion des aspérités. M. Schneider retrouve dans ses expériences le palier horizontal ayant donné lieu à la notion de tension critique. Pour le fer, par exemple, ce palier se poursuit dans certains cas jusqu'à une intensité de plusieurs ampères. Cette tension critique résulte donc de la compensation mutuelle des diverses causes de variation de la force électromotrice, elle n'est pas une constante naturelle. Ce phénomène n'est pas sans analogie partielle avec celui de l'arc électrique, dans lequel, par suite de l'agrandissement du cratère aux fortes intensités, la résistance diminue en même temps que l'intensité augmente. M. Schneider montre ce caractère accidentel de la tension critique en constatant que, dans ses nombreuses expériences, le fer et l'aluminium seuls donnent une portion de courbe bien horizontale. Il trouve pour le platine, l'or, l'argent, l'étain, le zinc, le plomb et le nickel les courbes dans lesquelles il y a encore une région sensiblement rectiligne, mais ascendante. Au contraire, pour un contact de deux surfaces de magnétite, il trouve une portion de courbe rectiligne descendante.

Un accroissement de pression initiale a pour effet d'augmenter l'étendue du contact. Il faudra donc une intensité plus grande pour amener la soudure et la variation de résistance qui lui est attribuée.

Si le contact est réalisé au moyen de barreaux se touchant bout à bout, plus le diamètre des barreaux sera fort, plus il y aura de chaleur enlevée par conductibilité. La variation de résistance se produira donc pour un courant plus faible avec des barreaux minces qu'avec des barreaux de fort calibre.

Ces deux conséquences sont vérifiées par l'expérience.

La $f g$. 2 représente le cohéreur-balance de $\mathbf{M}$. Schneider qui sert à mesurer l'adhérence entre les deux corps en contact. Un fléau très léger porte à l'une de ses extrémités un barreau vertical du 
corps à étudier. reposant par son extrémité sur l'extrémité d’un barreau identique. A lautre bout du fléau se trouve une petite pièce de fer attirée par un électro-aimant.

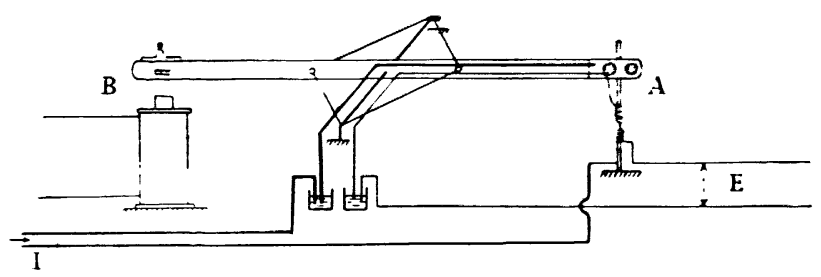

Firi. 2.

Latraction de cet électro-aimant itait au préalable étalonnée au milligramme près en fonction du courant qui y passe.

Les mesures ont montré que, dans l'intervalle qui correspond aux variations de résistance indiquées par la caractéristique, l'adhésion est sensiblement proportionnelle à l'inverse de la résistance, c’est-àdire la conductibilité du contact. M. Schneider trouve, par exemple, pour un contact platine-platine :

\begin{tabular}{|c|c|c|c|}
\hline Courant & $\begin{array}{l}\mathrm{P} \\
\text { Adhésion }\end{array}$ & lin & $P \times R$ \\
\hline $8, \underline{2}$ ampères & $3, \therefore 0 \div 1,1 \mathrm{mmes}$ & $0.01 \mathrm{iti}$ nhoms & $0,0.311$ \\
\hline$\tau, 0$ & $2,-2$ & $0,019,3$ & $\therefore .1$ \\
\hline 6,0 & 2,26 & $0,02+4$ & :3.:1 \\
\hline$\breve{3}, 0$ & $1,6^{\prime} \mathbf{x}$ & 0,0309 & ה \\
\hline 4,0 & 1,42 & 0,0394 & ร.:8 \\
\hline 3,0 & $0,9 \ddot{z}$ & $0,0: 314$ & 490 \\
\hline
\end{tabular}

Le fer a donné des résultats analogues.

Si la fusion est cause de la variation de résistance, celle-ci l,it -.. produire plus facilement quand un porte le contact à une tempuciture élevée au moyen d'une source de chaleur extricure. Liexpirience a été faite en entourant le contat' diune force électrique de petites dimensions, et elle a verifie len previ-inus.

L'élévation de température a pu itrenm-tatere directement enformant le contact de deux métanx dimirent- l pu mesur.unt leur force électromotrice thermo-électrique. () na trouve antw
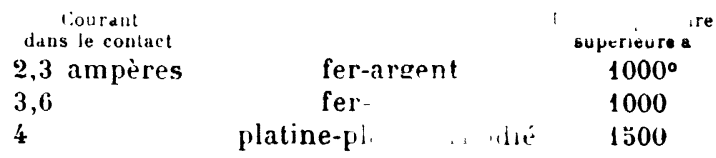

J. de Phys., 4' série, t. V. (Juillet 1906.) 
Cette élevation de température est très fugitive, elle disparait d'autant plus vite pour un mème contact qu'elle est obtenue avec un courant plus faible. M. Schneider attribue à cette circonstance la difficulté que l'on rencontre à construire un galvanomètre à indications assez rapides pour la mettre en évidence à de plus faibles intensités.

M. Schneider s'est préoccupé de rechercher la continuité des effets observés en partie avec des courants intenses avec ceux qui se produisent dans le cohéreur proprement dit.

- Avec un oscillateur de Lodge, placé à ä mètres de distance, dont il faisait varier le fonctionnement, il obtint des variations de résistance tout à fait analogues à celles qu'il avait provoquées par le passage direct du courant.

Il put aussi, au moyen de ces oscillations, provoquer des adhérences entre les métaux en contact, variant de $0^{\mathrm{gr}}, 02$ à $0^{\mathrm{gr}}, 7 \mathrm{ŏ}$.

M. Schneider avait cru pouvoir généraliser et attribuer les plıénomènes du cohéreur proprement dit à la même cause. Lextrapolation était un peu hardie. Les quantités d'énergie mises en œuvre dans ses expériences sont incomparablement plus grandes que celles qui agissent dans la télégraphie sans fil. On peut se demander s'il n'aurait pas imité, par une étude de laboratoire, la soudure électrique des rails plutôt que le cohéreur. Mais, si la résistance au point de contact de deux arètes vives est extrêmement grande, ou, si l'on veut, même lorsqu il n'y passe qu'un courant très faible, la densité du courant est énorme dans le voisinage de l'arête. Dans le cas des courants induits par des oscillations hertziennes, la conductibilité thermique n'a pas le temps d'intervenir pour enlever la chaleur de Joule. On doit donc admettre que de petites quantités de matières atteignent fréquemment des températures élevées. L’explication de M. Schneider conserve donc une certaine vraisemblance.

La très belle étude sur le cohéreur que M. Blanc a présentée à la Société de Physique, dans la séance du ỗ mai 190ð ('), nous a appris depuis qu'il existe au début de la cohération un phénomène caractéristique différent de la soudure, qui est la diffusion des couches de passage des corps en contact. M. Blanc a appuyé sa manière de voir sur des expériences très concluantes et l'a reliée à d'autres faits déjà connus, en apparence étrangers, au moyen d'une théorie fondée sur la théorie électronique de la conduction métallique.

(1) J. de Phys., 4e série, t. IV, p. 743; 190 s. 
L'on pourrait, il est vrai, établir sans peine une échelle continue de phénomenes intermédiaires entre la diffusion, telle que la conçoit M. Blanc, et la soudure. Mais cette continuité serait illusoire; M. Blanc a montré que lélévation de température n'est pas nécessaire à la cohération.

La soudure doit-elle néanmoins conserver une rertaine place dans d'explication des phénomènes dont les cinllw urs pratiquement employés sont le siège? Cela nest pas impossible. Remarquons que, dans les expériences de $\mathbf{l l}$. Blanc, toute- les ariffes vives sur lesquelles peut se localiser une gluantite de chateur in-t.tnt nit m. nt dégagée sont éritées, tandis que, dans la pratique. on a truuvé avantageux d'utiliser des fragments métalliques à arètes aussi vives que possible.

En revendiquant pour M. Schneider la part de vírité qui peut être contenue dans son explication, je ne crois pas diminuer le mérite de M. Blanc. Tandis que M. Schneider s'est contenté de tirer tout le parti possible de ce phénomine bien connu de la soudure ilectrique, M. Blanc a enrichi la Physique moléculaire de données nouvelles. 